\title{
Effect of age and sex on efficacy and tolerability of $\beta$ blockers in patients with heart failure with reduced ejection fraction: individual patient data meta-analysis
}

\author{
Dipak Kotecha, ${ }^{1,2}$ Luis Manzano, ${ }^{3}$ Henry Krum, ${ }^{2}$ Giuseppe Rosano, ${ }^{4,5}$ Jane Holmes, ${ }^{6}$ \\ Douglas G Altman, ${ }^{6}$ Peter D Collins, ${ }^{7}$ Milton Packer, ${ }^{8}$ John Wikstrand, ${ }^{9}$ Andrew J S Coats, ${ }^{10,11}$ \\ John G F Cleland, ${ }^{7}$ Paulus Kirchhof, ${ }^{1}$ Thomas G von Lueder, ${ }^{12}$ Alan S Rigby, ${ }^{13}$ Bert Andersson, ${ }^{9}$ \\ Gregory YH Lip, ${ }^{1}$ Dirk J van Veldhuisen, ${ }^{14}$ Marcelo C Shibata, ${ }^{15}$ Hans Wedel, ${ }^{16}$ Michael Böhm, ${ }^{17}$ \\ Marcus D Flather ${ }^{18}$ on behalf of the Beta-Blockers in Heart Failure Collaborative Group
}

For numbered affiliations see end of article.

Correspondence to: D Kotecha, University of Birmingham Institute of Cardiovascular Sciences, Medical School, Edgbaston, Birmingham B15 2TT, UK d.kotecha@bham.ac.uk Additional material is published online only. To view please visit the journal online.

Cite this as: $B M J$ 2016;353:11855 http://dx.doi.org/10.1136/bmj.i1855

Accepted: 14 March 2016

\section{ABSTRACT}

OBJECTIVES

To determine the efficacy and tolerability of $\beta$ blockers in a broad age range of women and men with heart failure with reduced ejection fraction (HFrEF) by pooling individual patient data from placebo controlled randomised trials.

\section{DESIGN}

Prospectively designed meta-analysis of individual patient data from patients aged $40-85$ in sinus rhythm at baseline, with left ventricular ejection fraction $<0.45$. PARTICIPANTS

13833 patients from 11 trials; median age 64; $24 \%$ women.

\section{MAIN OUTCOME MEASURES}

The primary outcome was all cause mortality; the major secondary outcome was admission to hospital for heart failure. Analysis was by intention to treat with an adjusted one stage Cox proportional hazards model.

RESULTS

Compared with placebo, $\beta$ blockers were effective in reducing mortality across all ages: hazard ratios were 0.66 ( $95 \%$ confidence interval 0.53 to 0.83 ) for the first quarter of age distribution (median age 50); 0.71 (0.58 to 0.87 ) for the second quarter (median age 60 ); 0.65 ( 0.53 to 0.78 ) for the third quarter (median age 68 ); and 0.77 (0.64 to 0.92 ) for the fourth quarter (median age 75). There was no significant interaction when age was modelled continuously $(\mathrm{P}=0.1)$, and the absolute reduction in mortality was $4.3 \%$ over a median follow-up of 1.3 years (number needed to treat 23). Admission to hospital for heart failure was significantly reduced by $\beta$

\section{WHAT IS ALREADY KNOWN ON THIS TOPIC}

$\beta$ blockers can reduce mortality and hospital admission in patients with heart failure with reduced ejection fraction and sinus rhythm

Older patients and women often receive less evidence based treatment and often at lower doses than shown to be effective in clinical trials

\section{WHAT THIS STUDY ADDS}

This study used individual patient data from all major randomised controlled trials comparing $\beta$ blockers with placebo in patients with heart failure with reduced ejection fraction and sinus rhythm

In these patients, treatment with $\beta$ blockers reduced all cause mortality and hospital admissions for heart failure, regardless of age or sex, and the tolerability of treatment was the same with $\beta$ blockers and placebo blockers, although this effect was attenuated at older ages (interaction $\mathrm{P}=0.05$ ). There was no evidence of an interaction between treatment effect and sex in any age group. Drug discontinuation was similar regardless of treatment allocation, age, or sex (14.4\% in those give $\beta$ blockers, $15.6 \%$ in those receiving placebo).

\section{CONCLUSION}

Irrespective of age or sex, patients with HFrEF in sinus rhythm should receive $\beta$ blockers to reduce the risk of death and admission to hospital.

\section{REGISTRATION}

PROSPERO CRD42014010012; Clinicaltrials.gov NCT00832442.

\section{Introduction}

$\beta$ blockers reduce morbidity and mortality in patients with heart failure and reduced left ventricular ejection fraction and are a cornerstone of modern evidence based treatment. Current heart failure guidelines do not differentiate treatment on the basis of age or sex, although initiation and maintenance of treatment is suboptimal both in older people and women. ${ }^{1-3}$ With increasing age, patients are more likely to be women (with less marked reduction in left ventricular ejection fraction). This interaction, along with relatively low numbers of older patients in randomised controlled trials, has created uncertainty about the optimum management of elderly patients with heart failure and reduced ejection fraction (HFrEF), both in women and men. Moreover, there are theoretical concerns about altered pharmacokinetics in older people that might affect the dose required or the tolerability of treatment. ${ }^{45}$ Although subgroup data and the results from SENIORS (Study of the Effects of Nebivolol Intervention on Outcomes and Rehospitalisation in Seniors with Heart Failure) suggest that the efficacy of $\beta$ blockers is retained in older patients, ${ }^{6-8}$ low uptake and poor maintenance of treatment continue to be a clinical reality.

The Beta-blockers in Heart Failure Collaborative Group was set up to combine individual patient data from major randomised controlled trials in heart failure and provide clear direction on clinically relevant subsets of patients in which there is uncertainty about the balance of safety and efficacy of $\beta$ blockers. ${ }^{910}$ Meta-analysis of individual patient data allows more robust examination of treatment effects in subgroups and enables of time to event analyses adjusted for baseline covariates, making it ideal for the appropriate pooling of original data. ${ }^{11}$ We have 
previously shown that morbidity and mortality are not improved by $\beta$ blockers in patients with HFrEF and concomitant atrial fibrillation, in contrast with patients in sinus rhythm, who had substantial reductions in admission to hospital and all cause mortality. ${ }^{12}$

In this analysis, we explored the interactions of $\beta$ blocker efficacy and tolerability with age and sex, using the largest and most robust dataset of pooled data from randomised trials. Our aim was to inform clinicians on the appropriate use of these important therapeutic agents for patients with HFrEF in sinus rhythm.

\section{Methods}

The Beta-blockers in Heart Failure Collaborative Group (BB-meta-HF) is a multinational effort, combining individual data from the major randomised controlled trials investigating the use of $\beta$ blockers in heart failure. The group consists of the leading investigators of these trials and international experts, with the support of the four pharmaceutical companies that conducted the original trials (AstraZeneca, GlaxoSmithKline, Merck Serono, and Menarini). This report was prepared according to the Preferred Reporting Items for Systematic reviews and Meta-Analyses of individual participant data (PRISMA-IPD) guidelines ${ }^{13}$ and prospectively registered with Clinicaltrials.gov (NCT0083244) and the PROSPERO database of systematic reviews (CRD42014010012). ${ }^{10}$ Detailed rationale and methods have previously been published. ${ }^{912}$

\section{Eligibility, search strategy, and data collection}

Published or unpublished randomised controlled trials were identified through computer aided searches (for example, Medline and Current Contents), scrutiny of reference lists of trials, trials registries, meeting abstracts, and review articles as well as discussion with group members and pharmaceutical manufacturers. We included randomised controlled trials that reported mortality as a primary outcome or part of a composite

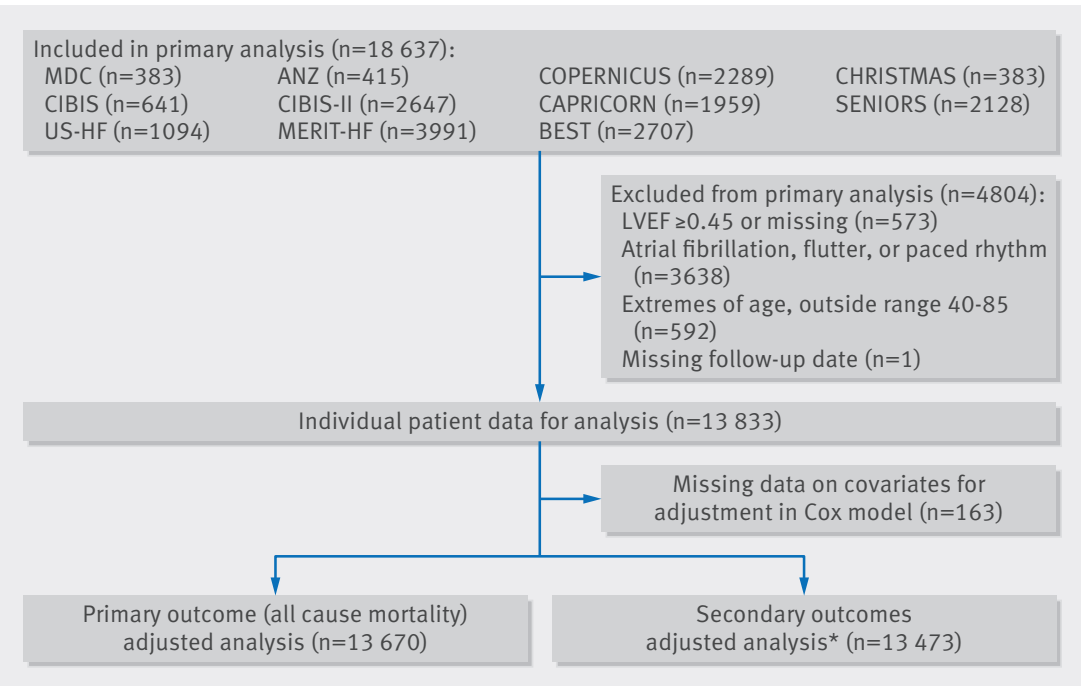

Fig 1 | Flowchart for included and excluded participants in analysis of effect of age and sex on efficacy and tolerability of $\beta$ blockers in patients with heart failure with reduced ejection fraction. $L V E F=$ left ventricular ejection fraction. * $M D C$ trial contributes only to mortality outcomes outcome comparing $\beta$ blockers versus placebo. Only unconfounded head to head trials were eligible, with recruitment of over 300 patients and planned follow-up of more than six months to make the project technically feasible and clinically relevant. The search results, individual study demographics and a standardised data request form to obtain individual patient data from each trial have been published. ${ }^{9}$

The 11 included studies account for $95.7 \%$ of eligible participants recruited in randomised controlled trials based on a systematic literature review: the Australia/ New Zealand Heart Failure Study (ANZ), ${ }^{14}$ the BetaBlocker Evaluation Survival Trial (BEST), ${ }^{15}$ the Carvedilol Post-Infarct Survival Control in LV Dysfunction Study (CAPRICORN), ${ }^{16}$ the Carvedilol Hibernating Reversible Ischaemia Trial: Marker of Success Study (CHRISTMAS), ${ }^{17}$ the Cardiac Insufficiency Bisoprolol Study (CIBIS I), ${ }^{18}$ the Cardiac Insufficiency Bisoprolol Study II (CIBIS-II), ${ }^{19}$ the Carvedilol Prospective Randomised Cumulative Survival Study (COPERNICUS), ${ }^{20}$ the Metoprolol in Idiopathic Dilated Cardiomyopathy Study (MDC), ${ }^{21}$ the Metoprolol CR/XL Randomised Intervention Trial in Congestive Heart Failure (MERIT-HF), ${ }^{223}$ the SENIORS Study, ${ }^{8}$ and the US Carvedilol Heart Failure Study (US-HF). ${ }^{24}$ All included studies had low overall risk of bias, as determined with the Cochrane Collaboration's risk of bias tool. ${ }^{25}$

\section{Patient involvement}

We have insufficient evidence to comment on whether patients were actively involved in the design or management of these 11 trials.

\section{Outcomes}

We extracted data from original source files, and additional follow-up mortality outcomes were available in seven studies. ${ }^{814-16202124}$ Our primary outcome was all cause mortality, including all reported deaths from each component study. Major secondary outcomes were all cause mortality during the trial period, all reported cardiovascular deaths, admission to hospital for heart failure, cardiovascular admission to hospital, fatal and non-fatal myocardial infarction, fatal and non-fatal stroke, and composites of mortality and hospital admission. One smaller study (1.4\% of patients) did not provide data on hospital admission or other adverse clinical events, ${ }^{21}$ though all studies contributed to the primary outcome (fig 1). Safety outcomes focused on discontinuation of study drug treatment because of adverse events (hypotension, bradycardia, exacerbation of heart failure, renal impairment, and respiratory dysfunction). We defined tolerability as the dose achieved as a percentage of maximum target dose, according to the particular $\beta$ blocker and specific trial design.

\section{Population}

Individual patient data were available for 18637 patients. For this analysis, restriction to patients with HFrEF was prespecified as a left ventricular ejection fraction cut off of $<0.45$, chosen to reflect the era in 
which these trials were undertaken, and the cardiac imaging distinction that separates patients with moderate and severe left ventricular dysfunction from those with mild or "intermediate" reduction in left ventricular ejection fraction. In light of our previous findings regarding the lack of prognostic benefit of $\beta$ blockers in patients with atrial fibrillation, we evaluated only those in sinus rhythm at baseline. ${ }^{12}$ To improve the robustness of the age analysis across the combined dataset, we also excluded the $4 \%$ of patients at the extremes of age (outside the age range of 40-85). One patient in the placebo arm was recorded as alive during study visits but had a missing final follow-up date and was excluded from analysis. Age was primarily assessed as a continuous variable, with prespecified division into quarters. Sex was explored as a secondary interaction variable across the age quarters of the age distribution.

\section{Statistical analysis}

Data are presented as percentages or medians and interquartile range (IQR; displayed as 25th-75th centiles). Estimated glomerular filtration rate was calculated with the modification of diet in renal disease (MDRD) formula, normalised to a body surface area of $1.73 \mathrm{~m}^{2}$. All analyses followed the principle of intention to treat. Outcomes were analysed with a stratified Cox proportional hazards regression model. ${ }^{26}$ This is a one stage fixed effects approach and assumes that all trials are estimating a common treatment effect with baseline hazards that vary across studies. Hazard ratios and 95\% confidence intervals are presented, along with corresponding $\mathrm{P}$ values. We prespecified adjustment in Cox models for age, sex, previous myocardial infarct, and baseline NYHA (New York Heart Association) class (I/II $v$ III/IV), left ventricular ejection fraction, heart rate, systolic blood pressure, diuretic therapy, and angiotensin converting enzyme inhibitors (ACEi) or angiotensin receptor blockers (ARB). Age was explored with numerous non-linear methods, including fractional polynomial models, but the best fit was obtained with a linear relation. As the amount of missing data for baseline characteristics was minimal, imputation was not required. The final population size for the fully adjusted Cox model for the primary outcome was 13670 (fig 1). We used Kaplan-Meier plots to graph the data (pooling data from all trials). As the follow-up periods in individual studies varied, data were censored at 1200 days (3.3 years) from randomisation. Heterogeneity for the primary outcome was assessed with $\chi^{2}$ test and $\mathrm{I}^{2}$ statistic, with the estimate of heterogeneity taken from the inverse variance fixed effects two stage model. ${ }^{29} \mathrm{We}$ performed a range of predefined sensitivity analyses, including alternative censor points, analysis of the entire age range, different left ventricular ejection fraction cut offs, exclusion of specific studies, additional baseline adjustment, and random effects modelling. ${ }^{28}$ Exploratory analyses included a per protocol assessment of patients who remained on study treatment throughout the trial. All models showed valid proportional hazards, as determined by Schoenfeld residuals. $^{29}$ Interactions were assessed in all models according to best practice..$^{3031}$ A two tailed $\mathrm{P}$ value of 0.05 was considered significant. Analyses were performed with Stata version 13.1 (StataCorp LP, TX) and R Version 3.0.2 (R Core Team, Vienna).

\section{Results}

\section{Age, sex, and baseline characteristics}

We included 13833 HFrEF patients in sinus rhythm in the analysis. The median age was 64 (IQR 55-71). Women accounted for $24 \%$ of patients overall $(\mathrm{n}=3283)$ and were older than men (66 (58-73) v 63 (55-71); fig A, appendix). The median duration of heart failure before enrolment was three years (one-six) and median left ventricular ejection fraction 0.27 (0.20-0.33). Table 1 shows baseline demographics according to age; table $\mathrm{A}$ in the appendix shows baseline demographics by sex.

\section{Primary outcome}

Overall $16 \%$ of patients died over a median follow-up period of 1.3 years (IQR 0.8-1.9). As expected, all cause mortality was higher in older patients, with relatively higher rates of death from heart failure than in younger patients (table B, appendix). Compared with men, women had lower absolute rates of all cause mortality (14\% $v 16 \%$ ), but causes of death were similar (table C, appendix).

$\beta$ blockers significantly reduced all cause mortality compared with placebo (968 deaths/7060 (13.7\%) $v$ $1222 / 6773(18.0 \%))$. The relative risk reduction with $\beta$ blockers was $24 \%$, with an absolute risk reduction of $4.3 \%$ (number needed to treat $23,95 \%$ confidence interval 18 to 32). The adjusted hazard ratio was 0.70 ( $95 \%$ confidence interval 0.64 to 0.77 ); $\mathrm{P}<0.001)$. Figure 2 shows the primary age analysis, assessing the hazard of death for $\beta$ blockers compared with placebo across the range of age, modelled as a continuous variable. No statistical interaction with age was identified $(\mathrm{P}=0.1$ for interaction). Table 2 displays the hazard ratio and figure 3 the Kaplan-Meier plots for each age quarter, confirming efficacy for the primary outcome in all quarters, including the oldest patients. Similar absolute risk reductions with $\beta$ blockers were noted in all age quarters (table $\mathrm{D}$ in appendix). $\beta$ blockers were effective in both women and men, in the whole group, and within specific age quarters (table 2 and fig 4). All sensitivity analysis for the primary outcome identified similar results to the main statistical model (table $\mathrm{E}$ in appendix).

\section{Secondary outcomes}

There was attenuation of the benefits of $\beta$ blockers on cardiovascular death by age $(\mathrm{P}=0.04$ for interaction) but there remained a significant reduction in events even in the oldest age group (table 3). Similar findings were seen with hospital admission for heart failure (fig 3 and table D in appendix), cardiovascular hospital admission and composite clinical outcomes. Fatal and non-fatal myocardial infarct or stroke were not reduced with $\beta$ blockers in the whole group nor in any age quartile (table 2). For all of the secondary outcomes, the effect in women and men were similar, with no interaction identified according to gender (table 2 and fig 4). 


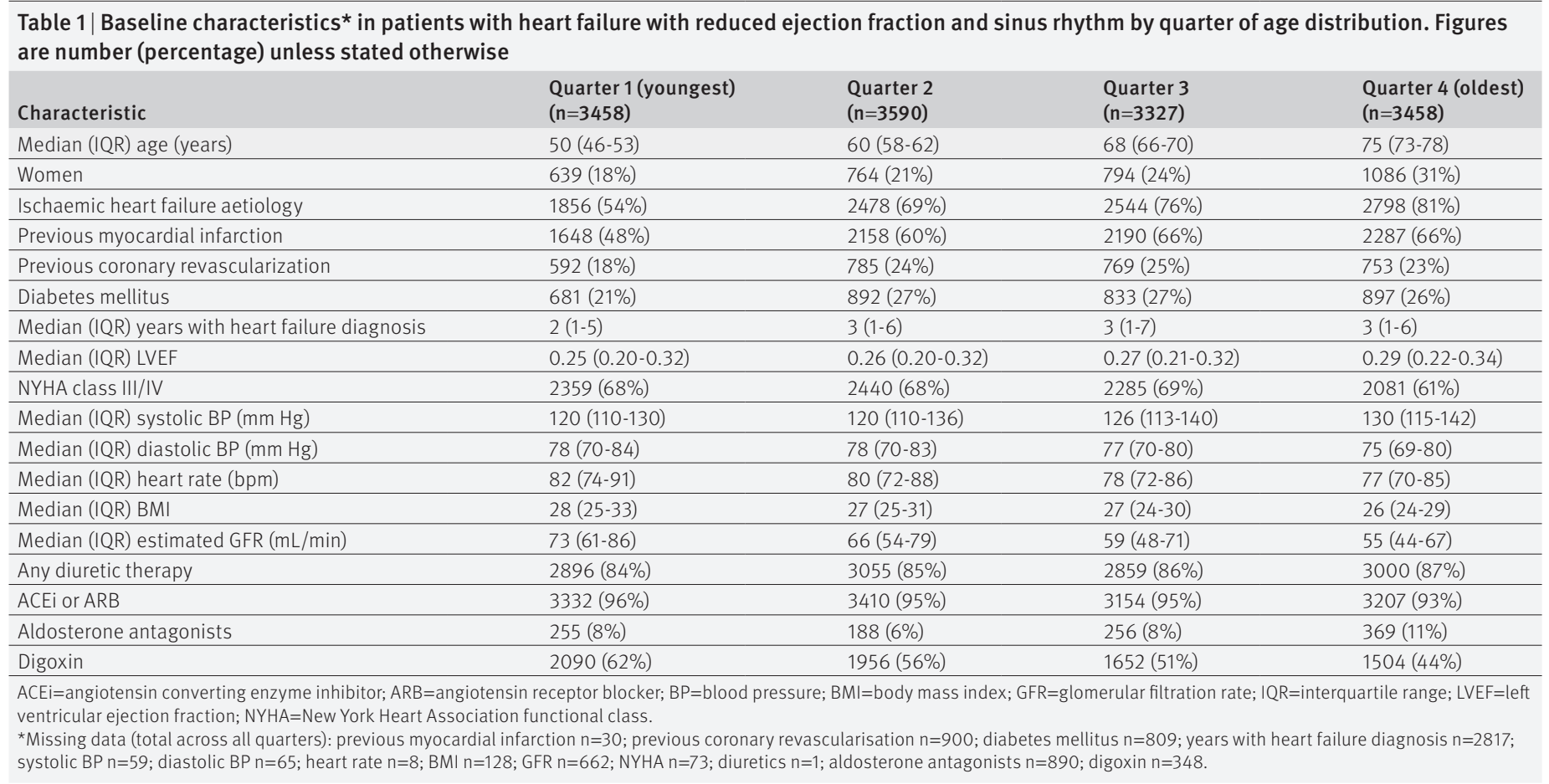

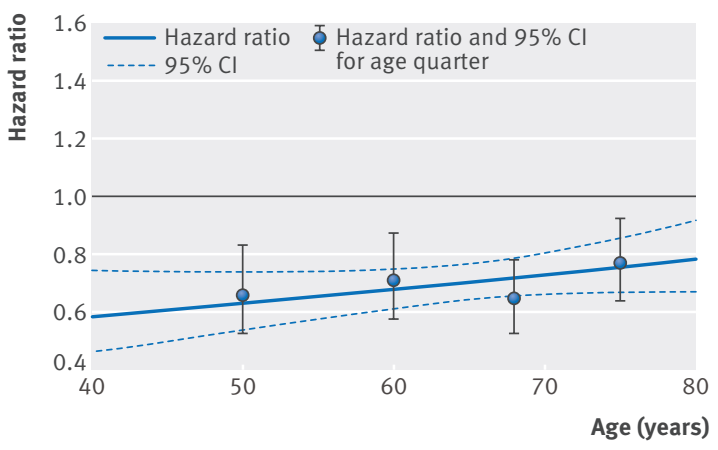

Fig 2 | $\beta$ blockers versus placebo hazard model for all cause mortality and age. Hazard ratio for $\beta$ blockers compared with placebo in HFrEF patients with sinus rhythm. Age modelled as continuous variable, with results for each quarter superimposed

Table 3 provides date on hospital admissions related to cardiovascular, events, all causes, and heart failure, divided by quarters of the age distribution and sex. Admission rates increased with age and were similar in both sexes. In the oldest quarter of age, patients had more than one admission per year, with half of these admissions because of heart failure, and a median length of stay of seven days in hospital.

\section{Tolerability of therapy}

There were similar rates of discontinuation because of adverse events in the placebo and $\beta$ blocker arms across quarters of the age distribution and sex, although these increased slightly with age (table 4). Overall, a numerically lower number of patients discontinued $\beta$ blockers (14.4\% $v 15.6 \%$ in placebo). Table $\mathrm{F}$ in the appendix shows specific causes of $\beta$ blocker discontinuation according to age and sex. Small numbers of patients discontinued treatment because of hypotension (0.7$1.6 \%$ ), bradycardia (0-3.5\%), exacerbation of heart failure (2.0-4.9\%), renal impairment (0-1.2\%), and respiratory compromise (0.5-1.2\%). Discontinuation was similar across age and sex, apart from a small excess in exacerbation of heart failure in the youngest age quarter for women compared with men $(5.4 \% \mathrm{v}$ $2.2 \%)$ and in bradycardia in the oldest men (3.5\% $v 0.7 \%$ in the oldest women). Dose of $\beta$ blocker did not differ across age and sex, with patients attaining $73 \%$ of the target dose at the interim study point (table $\mathrm{G}$ in appendix) compared with $84 \%$ achieving the corresponding dose of placebo.

\section{Discussion \\ Principal findings}

Using the near totality of available data from randomised controlled trials of $\beta$ blockers in patients with heart failure with reduced ejection fraction (HFrEF) and sinus rhythm, our analysis shows that there is no evidence of a clinically significant interaction with age or sex with respect to all cause mortality. We observed a significant benefit from $\beta$ blockers in each quarter of the age distribution, with absolute reductions in mortality of about $4 \%$ in the youngest and oldest patients. Results for hospital admission related to heart failure were similar, with significant reductions in each age quarter, albeit with minor attenuation of treatment effect in older patients. Discontinuation of treatment was similar in patients randomised to $\beta$ blockers or placebo, even in older patients, suggesting that "intolerance" of $\beta$ blockers in clinical practice could reflect false attribution to intercurrent events or preconceptions about side effects. 


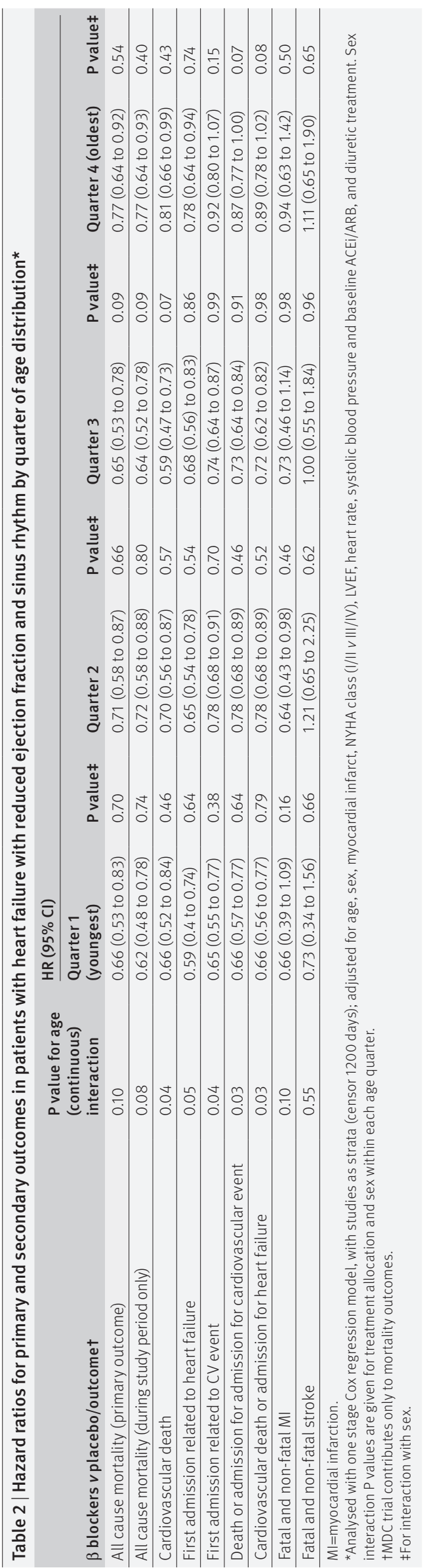

\section{Clinical context: age}

Heart failure guidelines recommend $\beta$ blockers for patients with HFrEF but have not previously been able to exclude an interaction with age. ${ }^{3233}$ As a result, prescription of treatment and long term continuation have been lower in older patients, ${ }^{34-36}$ presumably as clinicians trade off a perceived lower efficacy with other considerations such as potential adverse events and polypharmacy. The proportion of patients in our analysis aged $>70$ was $30 \%$, which does not reflect the "real world" population of HFrEF. In the three largest US heart failure registries (ADHERE, OPTIMIZE-HF, and Get With The Guidelines), the average age of patients with HFrEF was 70 (SD 14 and 14 and IQR 58-80, respectively; total 101066 patients). ${ }^{37}$ Similarly, in the Swedish Heart Failure Registry of 21864 patients with HFrEF, the mean age was 72 (SD 12). ${ }^{38}$ This confirms that older patients are under-represented in randomised controlled trials in heart failure, necessitating the pooling of data to provide information on treatment efficacy. In this context, individual patient data provide the only robust method to adequately combine subgroup data. ${ }^{39}$

There are important demographic changes noted with advancing age. Compared with the youngest quarter, older patients were more often women $(31 \% v 18 \%)$ and had more ischaemic aetiology ( $81 \% v 54 \%)$, higher left ventricular ejection fraction $(0.29 v 0.25)$, higher systolic blood pressure (130 $\mathrm{mm} \mathrm{Hg} v 20 \mathrm{~mm} \mathrm{Hg}$ ), lower heart rate ( $77 v 82$ beats $/ \mathrm{min}$ ), and reduced kidney function ( $55 v 73$ $\mathrm{mL} / \mathrm{min})$ and fewer received digoxin $(44 \% v 62 \%)$. These factors are known to affect prognosis in heart failure in different ways. Age is the most powerful predictor of prognosis but is not itself a predictor of response to treatment. The higher systolic blood pressure, higher left ventricular ejection fraction, and lower heart rate observed in older patients are predictors of better prognosis, while chronic renal impairment is associated with poor prognosis. The median length of heart failure before the trial was two years in the lowest age quarter and three years in the other age quarters, suggesting that patients were entered into the trials at a similar time after initial diagnosis, irrespective of age. Type of death also differed between age groups. Half of deaths in the youngest quarter are classified as "sudden" compared with 34\% in the oldest quarter, whereas deaths from heart failure accounted for $16 \%$ compared with $31 \%$, respectively. Thus the heterogeneity in age in the trials also reflects heterogeneity in aetiology of heart failure and comorbidity patterns. The results of this individual patient data meta-analysis, showing the clear benefit of $\beta$ blockers across all age groups despite this heterogeneity, is an important finding that cannot be predicted by analysis of patient characteristics and outcomes in observational datasets or mechanistic studies. Our assessment confirms that irrespective of different patient factors associated with age, patients in sinus rhythm benefit from treatment with a $\beta$ blocker, a novel and important finding that informs clinical practice and underpins the applicability of current clinical guidelines. ${ }^{3233}$

In general, the effect of $\beta$ blockers was consistent on the major secondary outcomes analysed. We did identify 


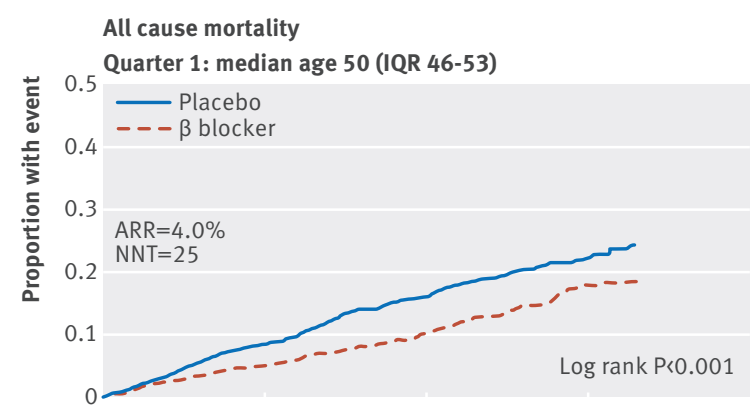

Numbers at risk

Placebo

$\begin{array}{cccc}1682 & 1117 & 407 & 183 \\ \begin{array}{c}\beta \text { blocker } \\ 1776\end{array} & 1263 & 517 & 261\end{array}$

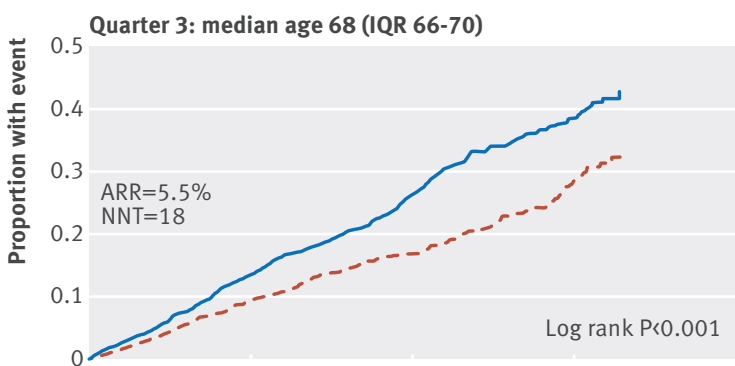

Numbers at risk

$\begin{array}{cccc}\begin{array}{c}\text { Placebo } \\ 1645\end{array} & 1011 & 285 & 102 \\ \begin{array}{c}10 \text { blocker } \\ 1682\end{array} & 1087 & 343 & 121\end{array}$

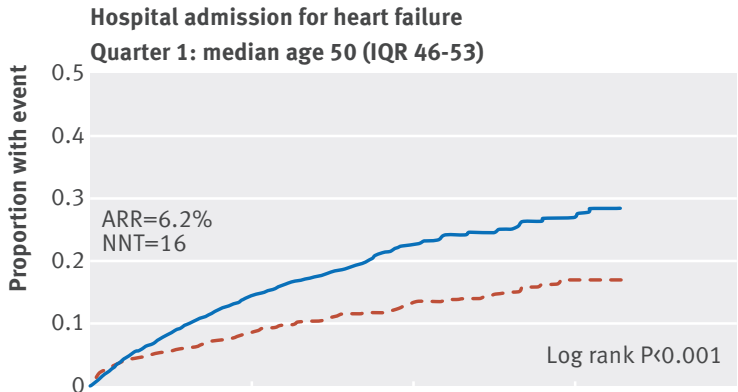

Numbers at risk

Placebo

Placebo
1627

$\beta$ blocker

1719

930

269

100

402

181

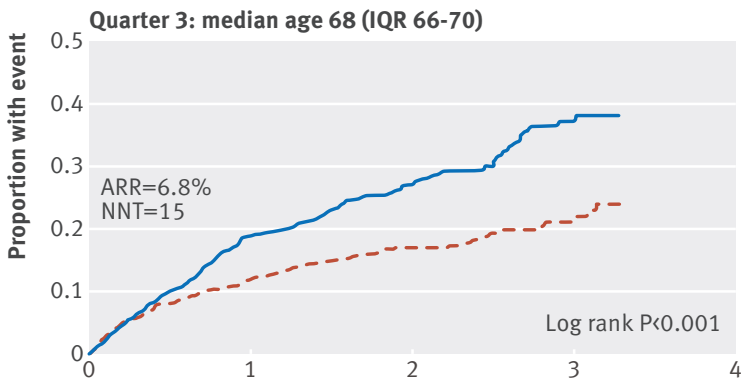

Numbers at risk

Placebo

$\begin{array}{cccc}1630 & 840 & 213 & 71 \\ \beta \begin{array}{c}\text { Bblocker } \\ 1668\end{array} & 976 & 282 & 95\end{array}$
Quarter 2: median age 60 (IQR 58-62)

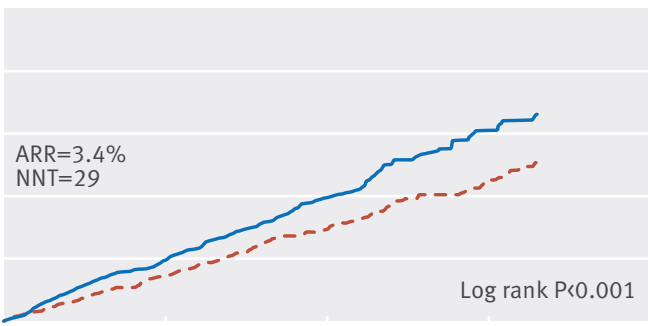

$\begin{array}{llll}1743 & 1129 & 353 & 127 \\ 1846 & 1238 & 441 & 187\end{array}$

Quarter 4: median age 75 (IQR 73-78)
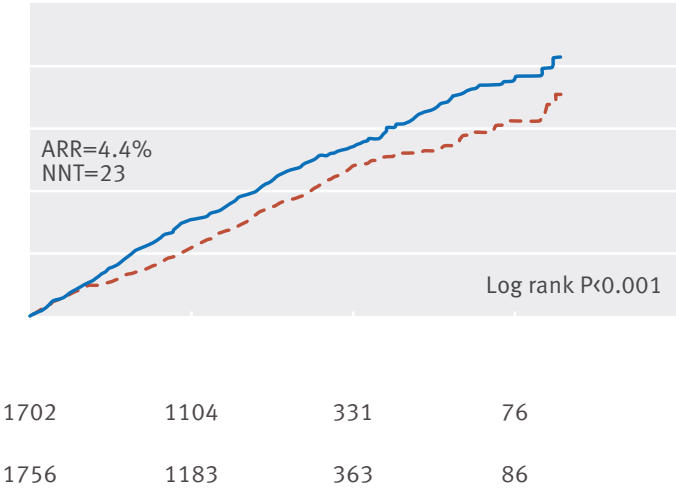

Quarter 2: median age 60 (IQR 58-62)

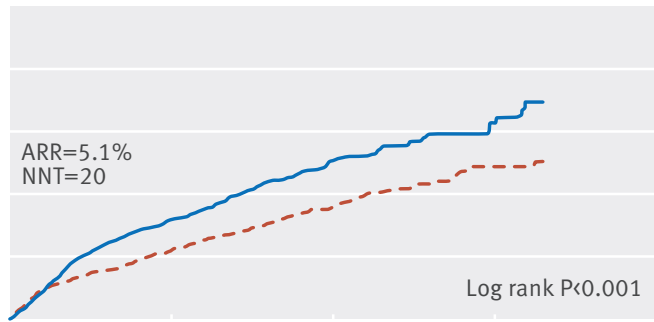

$\begin{array}{llll}1713 & 967 & 262 & 72 \\ 1814 & 1111 & 358 & 133\end{array}$

Quarter 4: median age 75 (IQR 73-78)

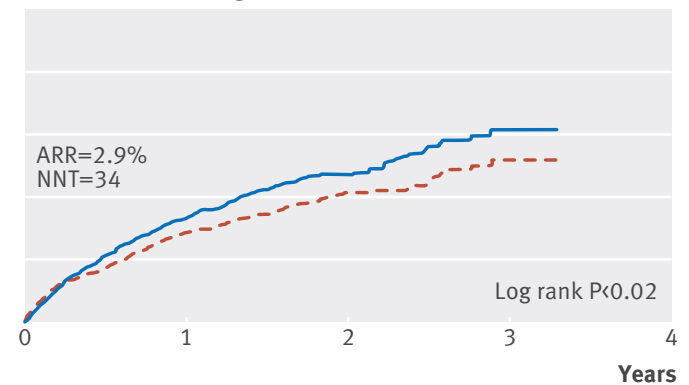

$\begin{array}{llll}1701 & 976 & 281 & 56 \\ 1756 & 1054 & 314 & 69\end{array}$

(admission for heart failure) age. $A R R=$ absolute risk reduction; $\mathrm{NNT}=$ number needed to treat 


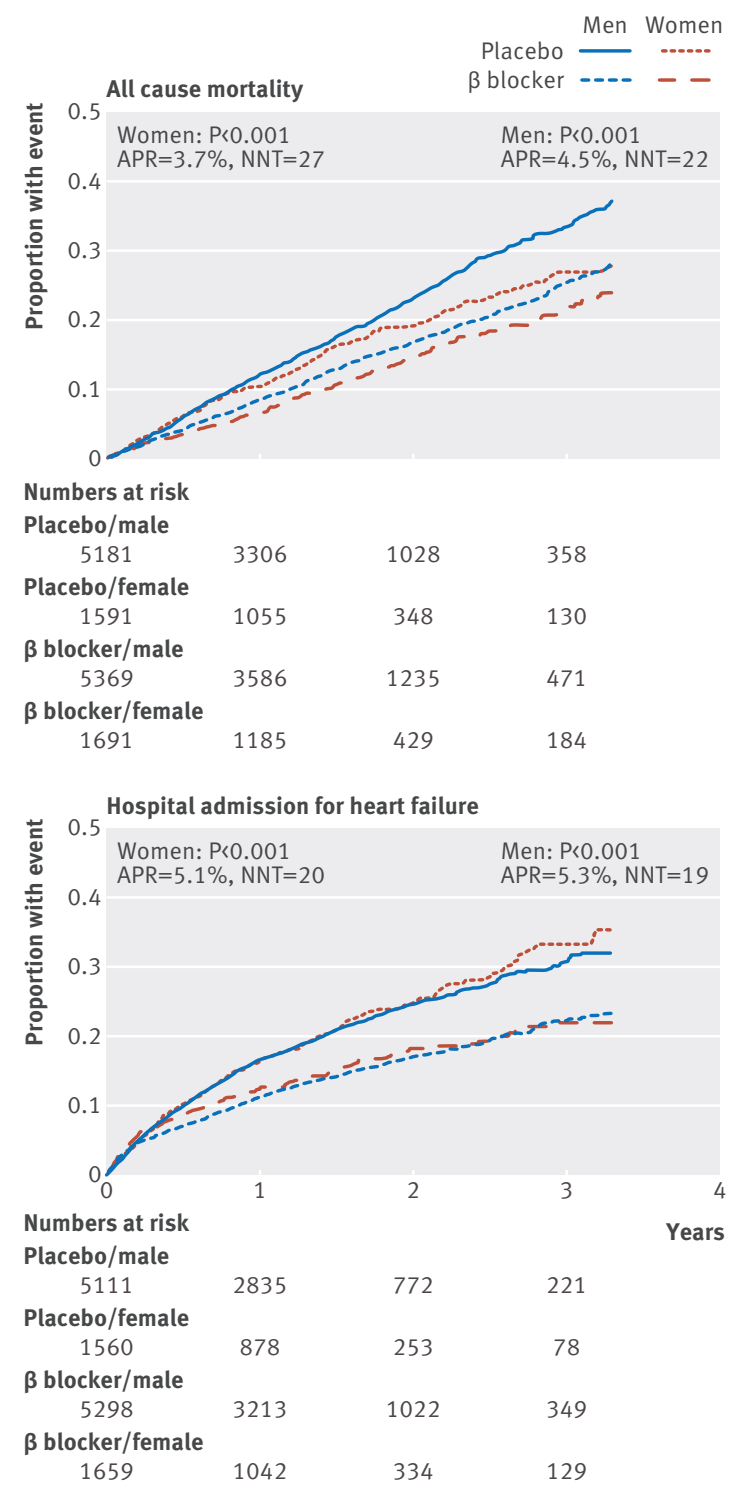

Fig 4 | Kaplan-Meier event curves according to sex for primary outcome (all cause mortality) and major secondary outcome (admission for heart failure) for $\beta$ blockers $v$ placebo by sex. $\mathrm{ARR}=$ absolute risk reduction; $\mathrm{NNT}=$ number needed to treat attenuated prevention of hospital admission by $\beta$ blockers with age $(\mathrm{P}=0.05$ for first hospital admission related to heart failure and $\mathrm{P}=0.04$ for first admission related to cardiovascular event). Adjusted hazard ratios, however, remained significant across age quarters and for all outcomes, apart from two secondary outcomes (hospital admission for cardiovascular event and the composite of cardiovascular death or admission for heart failure). Reassuringly, we did not identify differences in hospital admissions related to heart failure according to age or sex, and the length of hospital stay was similar in all patients, regardless of age or sex.

\section{Clinical context: sex}

Baseline demographics show that women with heart failure entered into randomised controlled trials tend to be older than men and differences such as higher systolic blood pressure, higher left ventricular ejection fraction, and lower estimated glomerular filtration rate might simply reflect this age difference. Women have a lower incidence of ischaemic aetiology and previous myocardial infarct, although a similar prevalence of heart failure compared with men. ${ }^{40}$ Prognosis has been shown to be better in women, though mortality rates are still 25\% over three years. ${ }^{41}$ Previous subgroup data from randomised controlled trials have provided conflicting results about the efficacy of $\beta$ blockers in women, including equal benefit (for example the MERIT-HF and CIBIS trials), ${ }^{42} 43$ enhanced mortality reduction compared with men (US Heart Failure trial), ${ }^{24}$ and also no effectiveness in women (BEST trial). ${ }^{44}$ Our results confirm that there is no difference in $\beta$ blocker efficacy in HFrEF according to sex. Cause of death in women and men showed identical proportions and patterns for sudden, heart failure, and non-cardiovascular deaths, which further support the concordance for recommendation of $\beta$ blockers. Thus, treatment with $\beta$ blockers should not be withheld from women with HFrEF, a practice that has been reported alongside fewer cardiology assessments and cardiac procedures. ${ }^{45}$ Women were under-represented in the clinical trials we analysed (24\%), and this continues to be the case-for

\begin{tabular}{|c|c|c|c|c|c|c|c|c|c|c|}
\hline \multirow[b]{2}{*}{ Admission type } & \multicolumn{2}{|c|}{ Quarter 1 (youngest) } & \multicolumn{2}{|l|}{ Quarter 2} & \multicolumn{2}{|l|}{ Quarter 3} & \multicolumn{2}{|c|}{ Quarter 4 (oldest) } & \multicolumn{2}{|l|}{ All ages } \\
\hline & Women & Men & Women & Men & Women & Men & Women & Men & Women & Men \\
\hline \multicolumn{11}{|l|}{ All cause } \\
\hline$\%$ with $\geq 1$ admission & $39 \%$ & $31 \%$ & $35 \%$ & $36 \%$ & $39 \%$ & $40 \%$ & $40 \%$ & $43 \%$ & $39 \%$ & $37 \%$ \\
\hline Average No (range) of admissions & $0.94(0-18)$ & $0.67(0-18)$ & $0.78(0-14)$ & $0.76(0-22)$ & $0.79(0-11)$ & $0.79(0-17)$ & $0.68(0-12)$ & $0.85(0-26)$ & $0.78(0-18)$ & $0.76(0-26)$ \\
\hline Annualised rate/patient & 0.67 & 0.69 & 0.85 & 0.81 & 0.87 & 0.86 & 1.05 & 1.06 & 0.85 & 0.85 \\
\hline \multicolumn{11}{|l|}{ Cardiovascular } \\
\hline$\%$ with $\geq 1$ admission & $25 \%$ & $20 \%$ & $23 \%$ & $25 \%$ & $27 \%$ & $27 \%$ & $28 \%$ & $30 \%$ & $26 \%$ & $25 \%$ \\
\hline Average No (range) of admissions & $0.48(0-13)$ & $0.38(0-14)$ & $0.44(0-13)$ & $0.46(0-16)$ & $0.47(0-10)$ & $0.46(0-10)$ & $0.43(0-12)$ & $0.50(0-16)$ & $0.45(0-13)$ & $0.44(0-16)$ \\
\hline Annualised rate/patient & 0.36 & 0.38 & 0.47 & 0.45 & 0.56 & 0.57 & 0.67 & 0.67 & 0.51 & 0.51 \\
\hline Average (IQR) length of stay (days) ${ }^{*}$ & $6(3-9)$ & $6(3-10)$ & $6(3-9)$ & $6(3-12)$ & $7(3-11)$ & $6(4-11)$ & $7(3-11)$ & $6(3-12)$ & $6(3-11)$ & $6(3-11)$ \\
\hline \multicolumn{11}{|l|}{ Related to heart failure } \\
\hline$\%$ with $\geq 1$ admission & $19 \%$ & $13 \%$ & $16 \%$ & $17 \%$ & $17 \%$ & $17 \%$ & $16 \%$ & $19 \%$ & $17 \%$ & $16 \%$ \\
\hline Average No (range) of admissions & $0.36(0-7)$ & $0.25(0-12)$ & $0.32(0-13)$ & $0.31(0-16)$ & $0.29(0-10)$ & $0.29(0-10)$ & $0.25(0-12)$ & $0.32(0-16)$ & $0.30(0-13)$ & $0.29(0-16)$ \\
\hline Annualised rate/patient & 0.24 & 0.27 & 0.32 & 0.30 & 0.33 & 0.35 & 0.46 & 0.45 & 0.34 & 0.34 \\
\hline Average (IQR) length of stay (days)* & $6(3-10)$ & $6(4-11)$ & $7(4-10)$ & $7(4-13)$ & $7(4-12)$ & $7(4-12)$ & $7(4-12)$ & $6(4-11)$ & $7(4-11)$ & $7(4-12)$ \\
\hline
\end{tabular}

*Based on first five hospital admissions for cardiovascular/heart failure cause. MDC trial does not contribute to admission outcomes. 


\begin{tabular}{|c|c|c|}
\hline $\begin{array}{l}\text { Discontinuation } \\
\text { because of any } \\
\text { adverse event }\end{array}$ & Placebo & $\beta$ blockers \\
\hline \multicolumn{3}{|c|}{ Quarter 1 (youngest) } \\
\hline Women & $51 / 300(17.0)$ & 47/335 (14.0) \\
\hline Men & $192 / 1375$ (14.0) & $167 / 1435(11.6)$ \\
\hline All & $243 / 1675$ (14.5) & 214/1770 (12.1) \\
\hline \multicolumn{3}{|l|}{ Quarter 2} \\
\hline Women & $53 / 366(14.5)$ & $51 / 394(12.9)$ \\
\hline Men & 201/1371 (14.7) & $183 / 1449$ (12.6) \\
\hline All & 254/1737 (14.6) & $234 / 1843(12.7)$ \\
\hline \multicolumn{3}{|l|}{ Quarter 3} \\
\hline Women & $59 / 383(15.4)$ & $59 / 411(14.4)$ \\
\hline Men & $205 / 1259(16.3)$ & $186 / 1271(14.6)$ \\
\hline All & $264 / 1642(16.1)$ & $245 / 1682(14.6)$ \\
\hline \multicolumn{3}{|c|}{ Quarter 4 (oldest) } \\
\hline Women & $90 / 537(16.8)$ & $86 / 549(15.7)$ \\
\hline Men & $200 / 1165(17.2)$ & $233 / 1207$ (19.3) \\
\hline All & 290/1702 (17.0) & $319 / 1756(18.2)$ \\
\hline \multicolumn{3}{|l|}{ All ages } \\
\hline Women & $253 / 1586(16.0)$ & $243 / 1689(14.4)$ \\
\hline Men & $798 / 5170(15.4)$ & $769 / 5362(14.3)$ \\
\hline All & $1051 / 6756(15.6)$ & $1012 / 7051(14.4)$ \\
\hline
\end{tabular}

example, in the recent large randomised controlled trial of angiotensin-neprilysin inhibition versus enalapril in HFrEF, women accounted for only $22 \%$ of the patients recruited. ${ }^{46}$ There is a clear need to improve the enrolment of women to provide realistic expectations of their risk and benefit from treatment.

Side effects and tolerability

Importantly for all ages and both sexes, we identified low rates of discontinuation of $\beta$ blockers because of adverse events and similar withdrawal rates to placebo. Although $\beta$ blockers are often associated with side effects, data from randomised trials consistently show no true difference compared with placebo in dizziness, diarrhoea, increased blood sugar concentration, or depression and little or no increase in lethargy with modern generation $\beta$ blockers. ${ }^{47-49}$ This information should reassure clinicians about the tolerability of $\beta$ blockers in view of the prognostic benefit we have identified in women and elderly people of both sexes. Elderly patients were able to reach similar maximal dose compared with younger patients with HFrEF.

\section{Strengths and limitations of study}

It is plausible that the benefits of $\beta$ blockers are attenuated in very old people (aged $>80$ ), although the amount of information on these patients from existing randomised controlled trials is scarce. Extrapolation from figure 2 indicates that any attenuation of prognostic benefit with age is actually quite mild and the effect of $\beta$ blockers in patients aged $>80$ will still be associated with hazard ratios of around 0.8 (giving a worthwhile $20 \%$ proportional reduction in the average risk of death). In the extreme elderly, it is worth noting that there are competing mortality risks. Even with pooling of individual patient data from all large datasets, there are limitations to inference of treatment effects in subgroups. Examination of treatment interactions by age shows significant treatment benefits in each age group, but assessment of interactions of age and sex will be limited by the size of the groups, number of events, and inclusion criteria for the component studies. For example, a lack of interaction might not provide full information on treatment effects in very elderly women because of the atypical elderly cohort included in the original trials. We specifically included only patients with HFrEF in sinus rhythm at baseline. Although sensitivity analyses including all patients showed a similar lack of interaction with age or sex, direct extrapolation to patients with left ventricular ejection fraction $>0.45$ cannot be made. Similarly, we have previously reported on outcomes for $\beta$ blockers in patients with concomitant heart failure and atrial fibrillation, ${ }^{12}$ and, although these patients have poor prognosis, ${ }^{50}$ they are distinctly different to patients with heart failure in sinus rhythm and require specific management. ${ }^{51}$

There have been changes in risk factor profiles and treatment patterns since the component studies were completed (for example, the use of cardiac resynchronisation treatment), however, $\beta$ blockers are still a vital component of optimal care in these patients ${ }^{52}$ and might have a synergistic effect (for example, with mineralocorticoid receptor antagonists)..$^{53}$ Finally, as with all meta-analytical techniques, we are limited by the data provided from the individual studies, with the inherent heterogeneity of patient populations. The strength of our analysis was the use of individual patient data from high quality randomised controlled trials, with near totality of available data and methodical data extraction from original datasets, ${ }^{9}$ resulting in improved quality of outcome data across trials.

\section{Conclusions and policy implications}

This analysis confirms that treatment with $\beta$ blockers reduces mortality and hospital admissions related to heart failure in patients with HFrEF with sinus rhythm, irrespective of age or sex. Absolute effect sizes for all cause mortality were similar across age quarters with no significant interaction when we used an adjusted continuous hazard model. In addition, the tolerability of $\beta$ blockers was similar to placebo, reinforcing the use of $\beta$ blockers in all patients with HFrEF in sinus rhythm and discouraging the practice of withholding such treatment in women or elderly patients.

\section{AUTHOR AFFILIATIONS}

'University of Birmingham Institute of Cardiovascular Sciences, Birmingham, UK

${ }^{2}$ Centre of Cardiovascular Research and Education in Therapeutics, Monash University, Melbourne, Australia

IInternal Medicine Department, Hospital Universitario Ramón y Cajal, Universidad de Alcalá, Madrid, Spain

${ }^{4}$ Department of Medical Sciences, IRCCS San Raffaele Pisana, Roma, Italy

${ }^{5}$ Cardiovascular and Cell Science Institute, St George's University of London, London, UK 
${ }^{6}$ Centre for Statistics in Medicine, Nuffield Department of Orthopaedics, Rheumatology and Musculoskeletal Sciences, University of Oxford, Oxford, UK

${ }^{7}$ National Heart and Lung Institute, Imperial College, London, UK ${ }^{8}$ Baylor University Medical Center, Dallas, USA

'Wallenberg Laboratory for Cardiovascular Research, Sahlgrenska Academy, Gothenburg University, Gothenburg, Sweden

${ }^{10}$ Monash Warwick Alliance, Monash University, Melbourne, Australia

${ }^{11}$ Monash Warwick Alliance, University of Warwick, Warwick, UK

${ }^{12}$ Department of Cardiology, Oslo University Hospital, Oslo, Norway

${ }^{13}$ Academic Cardiology, Castle Hill Hospital, Kingston upon Hull, UK

${ }^{14}$ Department of Cardiology, University Medical Centre Groningen, University of Groningen, Groningen, Netherlands

${ }^{15}$ Division of Cardiology, University of Alberta, Edmonton, Canada

${ }^{16}$ Nordic School of Public Health, Gothenburg, Sweden

${ }^{17}$ Universitätsklinikum des Saarlandes, Homburg/Saar, Germany

${ }^{18}$ Norwich Medical School, University of East Anglia, Norwich, UK

We are indebted to the other members of the Beta-blockers in Heart Failure Collaborative Group for database access and extraction support (for full list, please see design paper ${ }^{9}$ ), the steering committees of the included trials (in particular representatives of the MERIT-HF trial), as well as the late Philip Poole-Wilson (National Heart and Lung Institute, Imperial College, London, UK). This project was possible only with the support of the pharmaceutical companies that have marketed $\beta$ blockers in heart failure, and the group thanks AstraZeneca, GlaxoSmithKline, Menarini Farmaceutica, and Merck Serono for full access to trial data. We gratefully acknowledge incorporation of data from the BEST trial through the National Heart Lung and Blood Institute BioLINCC programme. This work is dedicated to the memory of Henry Krum (1958-2015).

Contributors: DK participated in the design of the study, manages the collaborative group and performed data management, statistical analysis, and manuscript preparation. LM, HK, and MDF participated in the design and coordination of the study and manuscript preparation. JH and DGA independently performed the primary statistical analyses. All named authors read, revised and approved the final manuscript. DK is guarantor.

Funding: BB-meta-HF was investigator initiated. Menarini Farmaceutica Internazionale also provided an unrestricted research grant for administrative costs and GlaxoSmithKline provided data extraction support. None of the pharmaceutical groups had any role in data analysis or manuscript preparation. DK is funded by a National Institute for Health Research (NIHR) Career Development Fellowship, although this work is not a direct output of the fellowship. The opinions expressed are those of the authors and do not represent the NIHR or the UK Department of Health.

Competing interests: All authors have completed the ICMJE uniform disclosure form (www.icmje.org/coi_disclosure.pdf) and declare: DK received grants from Menarini during the conduct of the study, and non-financial support from Daiitchi Sankyo, outside the submitted work. PDC has received lecture fees from Menarini, outside the submitted work. MP has received personal fees from AstraZeneca, Amgen, BioControl, Boston Scientific, Cardiokinetix, Cardiorentis, Cardio3, Novartis, Pfizer, and Takeda, all outside the submitted work. JW has received grants and personal fees from AstraZeneca during the conduct of the MERIT-HF study. AISC reports personal fees from Menarini during the conduct of the study but outside the submitted work. JGFC has received grants and personal fees from GlaxoSmithkline and personal fees from AstraZeneca, outside the submitted work. PK has received grants from British Heart Foundation, German Ministry of Education and Research, DZHK, Leducq

Foundation, several industry partners and personal fees from several industry companies, outside the submitted work. TGVL has received personal fees from Novartis and Vifor, outside the submitted work. BA has received grants and personal fees from AstraZeneca, during the conduct of the study; grants and personal fees from Servier, outside the submitted work. GYHL has served as a consultant for Bayer, Astellas, Merck, AstraZeneca, Sanofi, BMS/Pfizer, Biotronik, Portola, and Boehringer Ingelheim and has been on the speakers' bureau for Bayer, BMS/Pfizer, Boehringer Ingelheim, and Sanofi-Aventis, all outside the submitted work. HW has received personal fees from Astra-Zeneca, during the conduct of the study. MB has received personal fees from Servier, Boehringer Ingelheim, Medtronic, Bayer, during the conduct of the study but outside the submitted work.
Ethical approval: All original trials operated under supervision of an appropriate human ethics committee. The current analysis involves anonymised data only, hence ethics committee approval was deemed unnecessary by the National Research Ethics Service London.

Data sharing: No additional data are available, though details on statistical analysis are available from the corresponding author on request.

Transparency: The lead author (the manuscript's guarantor) affirms that this manuscript is an honest, accurate, and transparent account of the study being reported; that no important aspects of the study have been omitted; and that any discrepancies from the study as planned and registered have been explained.

This is an Open Access article distributed in accordance with the terms of the Creative Commons Attribution (CC BY 3.0) license, which permits others to distribute, remix, adapt and build upon this work, for commercial use, provided the original work is properly cited. See: http://creativecommons.org/licenses/by/3.0/.

1 Komajda M, Hanon O, Hochadel M, et al. Contemporary management of octogenarians hospitalized for heart failure in Europe: Euro Heart Failure Survey II. Eur Heart / 2009:30:478-86. doi:10.1093/eurheartj/ehn539.

2 Baumhäkel M, Müller U, Böhm M. Influence of gender of physicians and patients on guideline-recommended treatment of chronic heart failure in a cross-sectional study. EurJ Heart Fail 2009;11:299-303. doi:10.1093/eurjhf/hfn041.

3 Mehta PA, Cowie MR. Gender and heart failure: a population perspective. Heart 2006;92(Suppl 3):iii14-8. doi:10.1136/ hrt.2005.070342.

4 Ali Raza J, Movahed A. Use of cardiovascular medications in the elderly. Int / Cardiol 2002:85:203-15. doi:10.1016/ S0167-5273(02)00193-6

5 Hsich EM, Piña IL. Heart failure in women: a need for prospective data. J Am Coll Cardiol 2009;54:491-8. doi:10.1016/i.jacc.2009.02.066.

6 Dobre D, Haaijer-Ruskamp FM, Voors AA, van Veldhuisen DI. beta-Adrenoceptor antagonists in elderly patients with heart failure: a critical review of their efficacy and tolerability. Drugs Aging 2007:24:1031-44 doi:10.2165/00002512-200724120-00006.

7 Deedwania PC, Gottlieb S, Ghali JK, Waagstein F, Wikstrand JC. MERIT-HF Study Group. Efficacy, safety and tolerability of betaadrenergic blockade with metoprolol CR/XL in elderly patients with heart failure. Eur Heart / 2004:25:1300-9. doi:10.1016/j. ehj.2004.05.022.

8 Flather MD, Shibata MC, Coats AJ, et al. SENIORS Investigators. Randomized trial to determine the effect of nebivolol on mortality and cardiovascular hospital admission in elderly patients with heart failure (SENIORS). Eur Heart/ 2005;26:215-25. doi:10.1093/ eurheartj/ehi115.

9 Kotecha D, Manzano L, Altman DG, et al. Beta-Blockers in Heart Failure Collaborative Group. Individual patient data meta-analysis of beta-blockers in heart failure: rationale and design. Syst Rev 2013;2:7. doi:10.1186/2046-4053-2-7.

10 Kotecha D, Manzano L, Krum H, Altman DG, Holmes J, Flather M. The Beta-Blockers in Heart Failure Collaborative Group. Individual patient data meta-analysis. PROSPERO register. 2014: http://www.crd.york. ac.uk/PROSPERO/display_record.asp?ID=CRD42014010012.

11 Stewart LA, Tierney JF. To IPD or not to IPD? Advantages and disadvantages of systematic reviews using individual patient data. Eval Health Prof 2002;25:76-97. doi:10.1177/0163278702025001006.

12 Kotecha D, Holmes J, Krum H, et al. Beta-Blockers in Heart Failure Collaborative Group. Efficacy of $\beta$ blockers in patients with heart failure plus atrial fibrillation: an individual-patient data meta-analysis. Lancet 2014;384:2235-43. doi:10.1016/S0140-6736(14)61373-8.

13 Stewart LA, Clarke M, Rovers M, et al. PRISMA-IPD Development Group. Preferred Reporting Items for Systematic Review and Meta-Analyses of individual participant data: the PRISMA-IPD Statement. JAMA 2015;313:1657-65. doi:10.1001/jama.2015.3656.

14 Australia/New Zealand Heart Failure Research Collaborative Group. Randomised, placebo-controlled trial of carvedilol in patients with congestive heart failure due to ischaemic heart disease. Lancet 1997;349:375-80. doi:10.1016/S0140-6736(97)80008-6.

15 Beta-Blocker Evaluation of Survival Trial Investigators Beta-Blocker Evaluation of Survival Trial Investigators. A trial of the beta-blocker bucindolol in patients with advanced chronic heart failure. $N$ Engl/ Med 2001;344:1659-67. doi:10.1056/NEJM200105313442202.

16 Dargie HJ. Effect of carvedilol on outcome after myocardial infarction in patients with left-ventricular dysfunction: the CAPRICORN randomised trial. Lancet 2001;357:1385-90. doi:10.1016/ S0140-6736(00)04560-8

17 Cleland JG, Pennell DJ, Ray SG, et al. Carvedilol hibernating reversible ischaemia trial: marker of success investigators. Myocardial viability as a determinant of the ejection fraction response to carvedilol in patients with heart failure (CHRISTMAS trial): randomised controlled trial. Lancet 2003;362:14-21. doi:10.1016/S0140-6736(03)13801-9. 
18 A randomized trial of beta-blockade in heart failure. The Cardiac Insufficiency Bisoprolol Study (CIBIS). CIBIS Investigators and Committees. Circulation 1994:90:1765-73. doi:10.1161/01.CIR.90.4.1765.

19 The Cardiac Insufficiency Bisoprolol Study II (CIBIS-II): a randomised trial. Lancet 1999;353:9-13. doi:10.1016/S0140-6736(98)11181-9.

20 Packer M, Coats AJ, Fowler MB, et al. Carvedilol Prospective Randomized Cumulative Survival Study Group. Effect of carvedilol on survival in severe chronic heart failure. N Engl J Med 2001;344:1651-8. doi:10.1056/NEJM200105313442201

21 Waagstein F, Bristow MR, Swedberg K, et al. Metoprolol in Dilated Cardiomyopathy (MDC) Trial Study Group. Beneficial effects of metoprolol in idiopathic dilated cardiomyopathy. Lancet 1993:342:1441-6. doi:10.1016/0140-6736(93)92930-R.

22 Effect of metoprolol CR/XL in chronic heart failure: Metoprolol CR/XL Randomised Intervention Trial in Congestive Heart Failure (MERIT-HF). Lancet 1999:353:2001-7. doi:10.1016/S0140-6736(99)04440-2.

23 Hjalmarson A, Goldstein S, Fagerberg B, et al. MERIT-HF Study Group. Effects of controlled-release metoprolol on total mortality, hospitalizations, and well-being in patients with heart failure: the Metoprolol CR/XL Randomized Intervention Trial in congestive heart failure (MERIT-HF). JAMA 2000;283:1295-302. doi:10.1001/ jama.283.10.1295

24 Packer M, Bristow MR, Cohn JN, et al. U.S. Carvedilol Heart Failure Study Group. The effect of carvedilol on morbidity and mortality in patients with chronic heart failure. N Engl I Med 1996;334:1349-55. doi:10.1056/NEJM199605233342101.

25 Higgins JPT, Altman DG, Sterne JAC. Assessing risk of bias in included studies. In: Higgins JPT, Green S, eds. Cochrane Handbook for Systematic Reviews of Interventions Version 510. Cochrane Collaboration, 2011.

26 Tudur Smith C, Williamson PR. A comparison of methods for fixed effects meta-analysis of individual patient data with time to event outcomes. Clin Trials 2007;4:621-30. doi:10.1177/1740774507085276.

27 Smith CT, Williamson PR, Marson AG. Investigating heterogeneity in an individual patient data meta-analysis of time to event outcomes. Stat Med 2005;24:1307-19. doi:10.1002/sim.2050.

28 Schoenfeld D. Partial residuals for the proportional hazards regression model. Biometrika 1982;69:239-41. doi:10.1093/biomet/69.1.239.

29 Koopman L, van der Heijden GJMG, Hoes AW, Grobbee DE, Rovers MM. Empirical comparison of subgroup effects in conventional and individual patient data meta-analyses. Int I Technol Assess Health Care 2008;24:358-61. doi:10.1017/S0266462308080471.

30 Sauerbrei W, Royston P. Building multivariable prognostic and diagnostic models: transformation of the predictors by using fractional polynomials. JR Stat Soc 1999;162:71-94. doi:10.1111/1467-985X.00122.

31 Royston P, Sauerbrei W. A new approach to modelling interactions between treatment and continuous covariates in clinical trials by using fractional polynomials. Stat Med 2004;23:2509-25 doi:10.1002/sim.1815.

32 McMurray IJ, Adamopoulos S, Anker SD, et al. ESC Committee for Practice Guidelines. ESC Guidelines for the diagnosis and treatment of acute and chronic heart failure 2012: The Task Force for the Diagnosis and Treatment of Acute and Chronic Heart Failure 2012 of the European Society of Cardiology. Developed in collaboration with the Heart Failure Association (HFA) of the ESC. Eur Heart J 2012;33:1787-847. doi:10.1093/eurheartj/ehs104.

33 Yancy CW, Jessup M, Bozkurt B, et al. WRITING COMMITTEE MEMBERS American College of Cardiology Foundation/American Heart Association Task Force on Practice Guidelines. 2013 ACCF/AHA guideline for the management of heart failure: a report of the American College of Cardiology Foundation/American Hear Association Task Force on practice guidelines. Circulation 2013;128:e240-327. doi:10.1161/CIR.0b013e31829e8807.

34 Follath F. Beta-blockade today: the gap between evidence and practice. Eur Heart J Supp/ 2006;8(suppl C):C28-34doi:10.1093/eurheartj/sul011.

35 Shah SM, Carey IM, DeWilde S, Richards N, Cook DG. Trends and inequities in beta-blocker prescribing for heart failure. BrJ Gen Pract 2008;58:862-9. doi:10.3399/bjgp08X376195.

36 Sinha S, Goldstein M, Penrod J, et al. Brief report: beta-blocker use among veterans with systolic heart failure. J Gen Intern Med 2006:21:1306-9. doi:10.1111/j.1525-1497.2006.00601.x.
37 Mentz RJ, Kelly JP, von Lueder TG, et al. Noncardiac comorbidities in heart failure with reduced versus preserved ejection fraction. J Am Coll Cardiol 2014:64:2281-93. doi:10.1016/j.jacc.2014.08.036.

38 Alehagen U, Benson L, Edner M, Dahlström U, Lund LH. Association between use of statins and outcomes in heart failure with reduced ejection fraction: prospective propensity score matched cohort study of 21864 patients in the Swedish Heart Failure Registry. Circ Heart Fail 2015;8:252-60. doi:10.1161/CIRCHEARTFAILURE.114.001730.

39 Sun X, loannidis JP, Agoritsas T, Alba AC, Guyatt G. How to use a subgroup analysis: users' guide to the medical literature. JAMA 2014;311:405-11. doi:10.1001/jama.2013.285063.

40 Stromberg A, Martensson J. Gender differences in patients with heart failure. Eur / Cardiovasc Nurs 2003;2:7-18.

41 Martínez-Sellés M, Doughty RN, Poppe K, et al. Meta-Analysis Global Group In Chronic Heart Failure (MAGGIC). Gender and survival in patients with heart failure: interactions with diabetes and aetiology. Results from the MAGGIC individual patient meta-analysis. Eur I Hear Fail 2012;14:473-9. doi:10.1093/eurjhf/hfs026.

42 Leizorovicz A, Lechat P, Cucherat M, Bugnard F. Bisoprolol for the treatment of chronic heart failure: a meta-analysis on individual data of two placebo-controlled studies-CIBIS and CIBIS II. Cardiac Insufficiency Bisoprolol Study. Am Heart / 2002;143:301-7. doi:10.1067/mhj.2002.120768.

43 Ghali JK, Piña IL, Gottlieb SS, Deedwania PC, Wikstrand IC. MERIT-HF Study Group. Metoprolol CR/XL in female patients with heart failure: analysis of the experience in Metoprolol ExtendedRelease Randomized Intervention Trial in Heart Failure (MERIT-HF). Circulation 2002;105:1585-91. doi:10.1161/01. CIR.0000012546.20194.33.

44 Ghali IK, Krause-Steinrauf HJ, Adams KF, et al. Gender differences in advanced heart failure: insights from the BEST study. J Am Coll Cardiol 2003;42:2128-34. doi:10.1016/j.jacc.2003.05.012

45 Sheppard R, Behlouli H, Richard H, Pilote L. Effect of gender on treatment, resource utilization, and outcomes in congestive heart failure in Quebec, Canada. Am J Cardiol 2005;95:955-9. doi:10.1016/j. amjcard.2004.12.033.

46 McMurray JJ, Packer M, Desai AS, et al. PARADIGM-HF Investigators and Committees. Angiotensin-neprilysin inhibition versus enalapril in heart failure. N Engl J Med 2014;371:993-1004. doi:10.1056/ NEJMoa1409077.

47 Barron Al, Zaman N, Cole GD, Wensel R, Okonko DO, Francis DP. Systematic review of genuine versus spurious side-effects of beta-blockers in heart failure using placebo control: recommendations for patient information. Int / Cardiol 2013;168:3572-9. doi:10.1016/j.ijcard.2013.05.068.

48 Ko DT, Hebert PR, Coffey CS, et al. Adverse effects of beta-blocker therapy for patients with heart failure: a quantitative overview of randomized trials. Arch Intern Med 2004:164:1389-94. doi:10.1001/ archinte.164.13.1389.

49 Ko DT, Hebert PR, Coffey CS, Sedrakyan A, Curtis JP, Krumholz HM Beta-blocker therapy and symptoms of depression, fatigue, and sexual dysfunction. JAMA 2002;288:351-7. doi:10.1001/ jama.288.3.351

50 Kotecha D, Chudasama R, Lane DA, Kirchhof P Lip GY Atrial fibrillation and heart failure due to reduced versus preserved ejection fraction: A systematic review and meta-analysis of death and adverse outcomes. Int J Cardiol 2016;203:660-6. doi:10.1016/j.ijcard.2015.10.220.

51 Kotecha D, Piccini JP. Atrial fibrillation in heart failure: what should we do? Eur Heart / 2015:36:3250-7.

52 Voigt A, Shalaby A, Adelstein E, Saba S. Beta-blocker utilization and outcomes in patients receiving cardiac resynchronization therapy. Clin Cardiol 2010·33.E1-5 doi:10.1002/clc 20500.

53 Pitt B, Remme W, Zannad F, et al. Eplerenone Post-Acute Myocardial Infarction Heart Failure Efficacy and Survival Study Investigators. Eplerenone, a selective aldosterone blocker, in patients with left ventricular dysfunction after myocardial infarction. N Engl J Med 2003;348:1309-21. doi:10.1056/NEJMoa030207.

Appendix: Supplementary tables A-G and fig A 